\title{
The impact of COVID-19 pandemic lockdown on the incidence and outcome of complicated appendicitis
}

\author{
Yaron Rudnicki $^{1}$ (D) Hagai Soback ${ }^{1} \cdot$ Ori Mekiten $^{1} \cdot$ Guy Lifshiz $^{1} \cdot$ Shmuel Avital $^{1}$
}

Received: 18 March 2021 / Accepted: 20 July 2021 / Published online: 26 July 2021

(c) The Author(s), under exclusive licence to Springer Science+Business Media, LLC, part of Springer Nature 2021

\begin{abstract}
Background Patient attendance at emergency departments (EDs) during the COVID-19 pandemic outbreak has decreased dramatically under the "stay at home" and "lockdown" restrictions. By contrast, a notable rise in severity of various surgical conditions was observed, suggesting that the restrictions coupled with fear from medical facilities might negatively impact non-COVID-19 diseases. This study aims to assess the incidence and outcome of complicated appendicitis (CA) cases during that period.

Methods A retrospective study comparing the rate and severity of acute appendicitis (AA) cases during the COVID-19 initial outbreak in Israel during March and April of 2020 (P20) to the corresponding period in 2019 (P19) was conducted. Patient data included demographics, pre-ED status, surgical data, and postoperative outcomes.

Results Overall, 123 patients were diagnosed with acute appendicitis, 60 patients during P20 were compared to 63 patients in P19. The rate of complicated appendicitis cases was significantly higher during the COVID-19 Lockdown with 43.3\% (26 patients) vs. 20.6\% (13 patients), respectively ( $p<0.01)$. The average delay in ED presentation between P20 and P19 was 3.4 vs. 2 days $(p=0.03)$. The length of stay was 2.6 days in P20 vs. 2.3 days in P19 $(p=0.4)$, and the readmission rate was $12 \%$ (7 patients) vs. $4.8 \%$ (3 patients), $p=0.17$, respectively. Logistic regression demonstrated that a delay in ED presentation was a significant risk factor for complicated appendicitis (OR 1.139, CI 1.011-1.284).

Conclusion The effect of the COVID-19 initial outbreak and Lockdown coupled with hesitation to come to medical facilities appears to have discouraged patients with acute appendicitis from presenting to the ED as complaints began, causing a delay in diagnosis and treatment, which might have led to a higher rate of complicated appendicitis cases and a heavier burden on health care systems.
\end{abstract}

Keywords COVID-19 pandemic $\cdot$ Acute appendicitis $\cdot$ Complicated appendicitis $\cdot$ Lockdown $\cdot$ Stay at home

The Coronavirus disease-19 (COVID-19) was first reported in December 2019 in Wuhan, Hubei province, China [1]. During the following months, the contagion spread and developed into a global pandemic that reached the western hemisphere in the winter and spring of 2020 [2]. As the pandemic reached Israel in March 2020, similar to many governments around the world, rigid "stay at home" restrictions, followed by a nationwide "lockdown", were issued [3]. As expected, these restrictions had a significant impact on daily life with daily activities, such as work, social life,

Yaron Rudnicki

yaron217@gmail.com

1 Department of Surgery B, Meir Medical Center, Affiliated with the Sackler Faculty of Medicine, Tel-Aviv University, 4428164 Kfar Saba, Israel and leisure time, which were greatly limited, and the spread of COVID-19 was slowed down. On the other hand, another suspected consequence of these restrictions was a substantial reduction in healthcare attendance. An $80 \%$ reported decline in routine screening appointments for cervical, breast, and colon cancer in March and April of 2020 [4]. A decrease of more than $30 \%$ in emergency department (ED) visits of patients with non-trauma surgical complaints was also noted during that period [5]. In conjunction with the reduction in healthcare attendance and ED visits, there was a notable rise in the severity of various surgical conditions upon ED presentation [6-9].

One can hypothesize that the social concern of contracting COVID-19, propagated by the Lockdown and the following restrictions, resulted in delayed diagnosis in many cases and consequently, a higher complication rates in common 
surgical conditions. Acute Appendicitis (AA) and its variant Complicated Appendicitis were chosen as a test case. AA is among the most common causes of lower abdominal pain leading patients to present to the emergency department [10]. Gomes Laparoscopic Grading System of AA, which classifies appendicitis into five laparoscopic grades according to gross morphologic findings during surgical laparoscopy, was used. Grades 1 and 2 are considered noncomplicated appendicitis, while grades 3,4 , and 5 are considered complicated appendicitis [11].

This study aims to assess the incidence and outcome of complicated appendicitis cases during the COVID-19 outbreak social restrictions period in March and April of 2020 (P20) by comparing it to the corresponding period of March and April of 2019 (P19) in Israel and try to provide information on whether the "stay at home" restrictions had any adverse effects on the severity or outcomes of AA.

\section{Materials and methods}

A retrospective study was designed and performed to compare the rate, severity, and outcome of patients with complicated appendicitis during the Lockdown and social restrictions that were mandated in Israel during March and April 2020 at the height of the COVID-19 pandemic outbreak with reference to the corresponding period of March and April 2019. All patients diagnosed with acute appendicitis or a peri-appendicular disease at the emergency department of a single academic medical center in Israel during the periods mentioned above were included in this study.

The diagnosis was based on characteristic complaints, a surgeon's physical examination, inflammatory markers in the blood tests, including elevated white blood cells count (WBC) and elevated C-reactive protein (CRP) levels, followed by imaging, mainly abdominal double-contrast (oral and iv)-computerized tomography (CT) scan, abdominal ultrasound (US) exam, or both. The diagnosis was documented according to the international classification of disease, ninth edition, clinical modification (ICD-9) format [12]. The collected data included demographic features such as age, gender, body mass index (BMI), comorbidities, American Society of Anesthesiologists (ASA) score, duration of symptoms prior to presenting to the emergency department, and treatment approach (surgical or non-surgical). Clinical presentation, imaging, surgical findings, treatment, postoperative complications, and post-discharge follow-up were assessed to determine the severity of the appendicitis and its outcome. Postoperative pathology was reviewed to exclude patients with any diagnosis other than acute appendiceal inflammation, excluding mainly appendiceal neoplasm or a normal appendix.
The pre-intervention severity of inflammation was determined based on physical examination, inflammatory blood markers, and imaging findings such as appendiceal diameter, abscess formation, and the presence of an appendiceal fecalith. The characterization of a complicated appendicitis was made retrospectively based on surgical findings according to the Gomes Laparoscopic Grading System of Acute appendicitis [11] or the need for percutaneous drainage. The outcome was measured by the rate of intraoperative and postoperative complications, length of hospital stay (LOS), the need for postoperative intravenous (IV) or oral (PO) antibiotic treatment, the rate of readmissions, the intervention method upon readmission, and Clavien-Dindo classification for postoperative complications [13].

Statistical analyses were performed using SPSS $®$ version 25 package for Mac (SPSS Inc.). For comparison between the P19 and P20 groups, categorical variables were analyzed with Chi-square test, and continuous variables were analyzed with $t$ tests. Logistic regression analyses were conducted to evaluate the effect of pre-ED symptom duration on the rate of complicated appendicitis, controlling for various factors. For all tests, a $p$-value of less than 0.05 was considered significant.

The study was approved by the Institutional Review Board (Approval No MMC-0223-20) and was registered in ClinicalTrials.gov (ID - NCT04786041).

\section{Results}

Overall, 123 patients were diagnosed with acute appendicitis, 63 patients during P19 were compared to 60 patients in P20. The two groups were similar in most demographic features, such as mean age of 38.3 in P19 vs. 40.3 in P20 $(p=0.54)$, mean BMI 25.6 vs. 25.5 , and a relatively low percentage of patients with at least one comorbidity ( $21 \%$ vs. $35 \% p=0.077)$ and a high percentage of patients with an ASA score of only 1 or 2 (95\% vs. $85 \% p=0.09$ ), respectively. Additionally, 65\% (41 patients) were male in P20 compared to $42 \%$ (25 patients) in P19.

Upon presenting to the emergency department with abdominal complaints, patients reported an average duration of symptoms of 3.4 days in P20 compared to 2 days in $\mathrm{P} 19(p=0.03)$ prior to attending the ED (Fig. 1A). The difference in the inflammatory blood markers, white cell count (WBC), or in C-Reactive Protein level (CRP) between $\mathrm{P} 19$ and P20 was not significant (12.2 vs. $13.2, p=0.17$ and 5.5 vs.6.2, $p=0.64$, respectively). There was also no difference in the choice of abdominal imaging with most patients undergoing an abdominal double-contrast material CT (P19: 78\% vs. P20: 67\%, $p=0.17$ ) and the rest who underwent an ultrasound examination. Imaging of $21 \%$ of patients in P20 exhibited peri-appendicular findings such as 

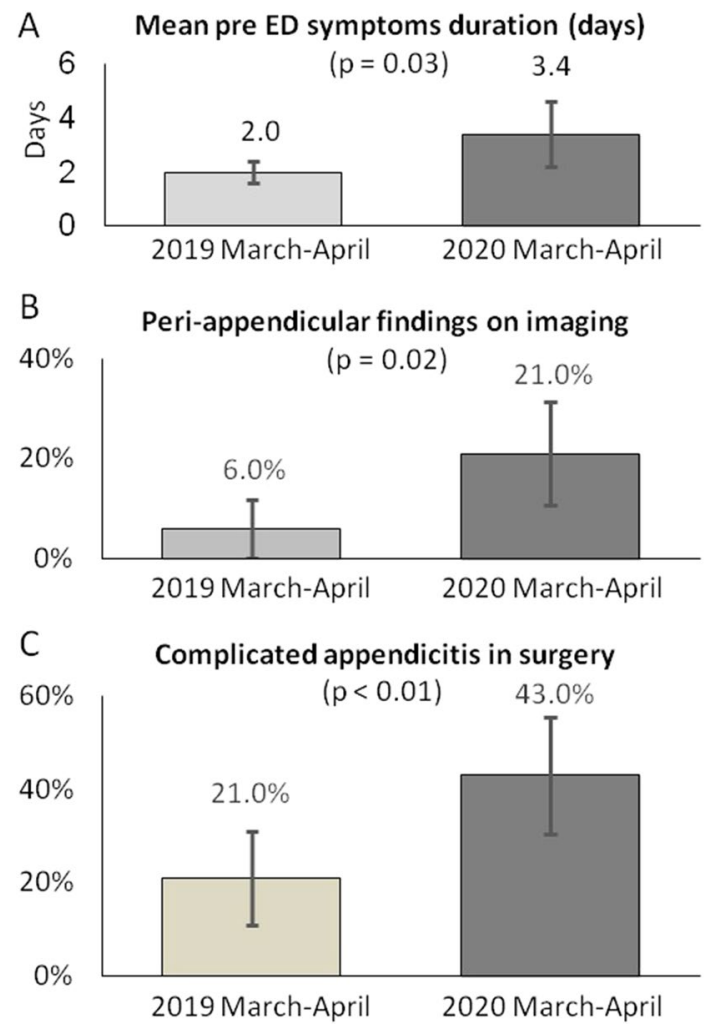

Fig. 1 A A comparison of the mean duration of pre-emergency department (ED) symptoms in days between the P19 and P20 groups. B A comparison of the percentage of cases with peri-appendicular findings on imaging (CT and US) between P19 and P20 groups. C A comparison of the percentage of cases with complicated appendicitis found in surgery between P19 and P20 groups

a peri-appendicular abscess or a peri-appendicular phlegmon compared to $6 \%$ of similar findings in P19 $(p=0.02)$ (Fig. 1B) impaling a more complicated disease (Table 1). The rate of patients with an appendiceal fecalith on imaging was fairly similar (P20: $18 \%$ vs. P19: $13 \%, p=0.39)$, as was the mean maximal appendiceal diameter on imaging (P20: $12 \mathrm{~mm}$ vs. P19: $11.8 \mathrm{~mm}, p=0.82$ ).

\section{Surgical findings and treatment}

The treatment approach was mainly a laparoscopic appendectomy (P20: 85\% vs. P19: 87\%, $p=0.71$ ). Open appendectomy was performed in 5\% of cases in P20 and 6\% in $\mathrm{P} 19(p=0.75)$, and 5\% in both $\mathrm{P} 20$ and P19 were treated with antibiotics alone. In P20, 5\% of patients were treated with a percutaneous drain and antibiotics for a peri-appendicular abscess, whereas none were treated that way in P19 $(p=0.08)$.

The rate of complicated appendicitis cases was $43.3 \%$ (26 patients) during the COVID-19 lockdown period of $\mathrm{P} 20$ compared to $20.6 \%$ (13 patients) in P19 $(p<0.01)$ (Fig. 1C). The rate of each type of complicated appendicitis is given in Table 2. Phlegmonous appendicitis cases were significantly higher in P20 with $13 \%$ of cases compared to $3 \%$ in P19 $(p=0.04)$, as were the cases of localized pus around the necrotic appendix with $13 \%$ in P20 compared to $2 \%$ in P19 $(p=0.02)$. There was no difference in intraoperative complications between the groups. Abdominal fluid for bacterial culture was taken in $25 \%$ of appendectomies in P20 compared to $10 \%$ in P19 $(p=0.03)$. Although the median lengths of operations were reasonably similar, $45 \mathrm{~min}$ in P19 vs. 46 min in P20, the number of operations that took longer than 90 min was higher in P20 with 10 cases (17\%) vs. 4 cases $(7 \%)$ in $\mathrm{P} 19(p=0.1)$.

\section{Clinical outcome}

The average length of hospital stay (LOS) was 2.6 days in P20 compared to 2.3 days in P19 $(p=0.42)$. Postoperative IV antibiotics were given to $47 \%$ of patients in P20 compared with $32 \%$ in P19 $(p=0.09)$ for varying durations of 3 to 10 days based on the severity of inflammation. Postoperative complications such as surgical site infections (SSI) were noted in $4 \%$ of patients in P20 and none in P19. The 30-day readmission rate was $12 \%$ (7 patients) in P20 compared to $5 \%$ (3 patients) in $\mathrm{P} 19(p=0.17)$. Out of the seven patients readmitted in P20, three patients necessitated a diagnostic laparoscopy and drainage. One patient received a percutaneous abdominal drain. All re-interventions were based on the surgical team's re-assessment of the patient's clinical condition and imaging study, and the decision was made accordingly. None of the three readmitted patients in P19 needed anything beyond antibiotic treatment. The average length of stay in the second admission was six days in P20 compared to 2.7 days in P19. The overall clinical follow-up time had a mean of 301 days for patients in P20 compared to 129 days in P19 (Table 3).

Logistic regression of all patients in P19 and P20 combined was performed to identify risk factors to develop a complicated appendicitis. The duration of symptoms prior to presenting to the emergency department was found to be a significant risk factor for complicated appendicitis (OR 1.139, CI 1.011-1.284) while controlling for age (OR 1.029, CI 1.006-1.052) and BMI (OR 1.102, CI 1.005-1.209) (Table 4).

\section{Discussion}

The severity of many surgical and infectious diseases is believed to directly correlate with the duration of the pretreatment period, and a delay in treatment initiation may lead to severe complications [14]. As shown in this dataset, the length of pre-ED symptoms duration and the time to medical and surgical intervention correlated with the severity of 
Table 1 Baseline demographic and clinical and imaging characteristics of patients

\begin{tabular}{|c|c|c|c|}
\hline Characteristic & $\begin{array}{l}\text { March-April } 2019 \\
\text { group }(n=63)\end{array}$ & $\begin{array}{l}\text { March-April } 2020 \\
\text { group }(n=60)\end{array}$ & $p$ Value \\
\hline Age $(y r)-$ mean $\pm S D$ & $38.3 \pm 17.3$ & $40.3 \pm 18.9$ & 0.54 \\
\hline Male sex-n (\%) & $41(65 \%)$ & $25(42 \%)$ & $<0.01$ \\
\hline $\mathrm{BMI}-$ mean $\pm \mathrm{SD}$ & $25.6 \pm 4.7$ & $25.5 \pm 4.8$ & 0.89 \\
\hline $1 \leq$ Co-Morbidities - $\mathrm{n}(\%)$ & $13(21 \%)$ & $21(35 \%)$ & 0.077 \\
\hline ASA class I or II-n (\%) & $60(95 \%)$ & $51(85 \%)$ & 0.086 \\
\hline Pre-ED symptoms duration (days) mean \pm SD & $2 \pm 1.6$ & $3.4 \pm 4.8$ & 0.03 \\
\hline $\mathrm{WBC}$ mean $\pm \mathrm{SD}$ & $12.2 \pm 4.4$ & $13.2 \pm 3.8$ & 0.17 \\
\hline $\mathrm{CRP}$ mean $\pm \mathrm{SD}$ & $5.5 \pm 8.6$ & $6.2 \pm 7.7$ & 0.64 \\
\hline \multicolumn{4}{|l|}{ Type of ED imaging-n (\%) } \\
\hline Abdominal CT & $49(78 \%)$ & $40(67 \%)$ & 0.17 \\
\hline Abdominal US & $12(19 \%)$ & $13(22 \%)$ & 0.72 \\
\hline Abdominal US + CT & $2(3 \%)$ & $7(12 \%)$ & 0.08 \\
\hline Peri-appendicular findings on imaging-n $(\%)$ & $4(6 \%)$ & $13(21 \%)$ & 0.02 \\
\hline Peri-appendicular abscess-n (\%) & 0 & $9(15 \%)$ & $<0.01$ \\
\hline Peri-appendicular phlegmon-n (\%) & $3(5 \%)$ & 0 & 0.08 \\
\hline Appendiceal fecalith on imaging- $\mathrm{n}(\%)$ & $8(13 \%)$ & $11(18 \%)$ & 0.39 \\
\hline Appendix diameter on imaging $(\mathrm{mm})-$ mean $\pm \mathrm{SD}$ & $11.8 \pm 4$ & $12 \pm 5.6$ & 0.82 \\
\hline
\end{tabular}

$B M I$ Body mass index - Weight in kilograms divided by the square of the height in meters, ASA American Society of Anesthesiologists, ED Emergency Department, WBC White Blood Cell, CRP C-Reactive Protein, CT Computed Tomography, US Ultrasound acute appendicitis. Most of the complicated appendicitis cases waited for more than two days before seeking medical treatment in P20 and P19. More than twice as many cases of complicated appendicitis during March and April of 2020 (P20) under the COVID-19 Lockdown, compared to the corresponding months of March and April of 2019 (P19), may
Table 2 Finding in surgery or non-operative treatment

\begin{tabular}{|c|c|c|c|}
\hline Characteristic & $\begin{array}{l}\text { March-April } \\
2019 \text { group } \\
(n=63)\end{array}$ & $\begin{array}{l}\text { March-April } \\
2020 \text { group } \\
(n=60)\end{array}$ & $p$ Value \\
\hline \multicolumn{4}{|l|}{ Intervention approach } \\
\hline Laparoscopic Appendectomy-n (\%) & $55(87 \%)$ & $51(85 \%)$ & 0.71 \\
\hline Open Appendectomy-n (\%) & $4(6 \%)$ & $3(5 \%)$ & 0.75 \\
\hline Laparoscopy converted to Open Appendectomy-n (\%) & $1(2 \%)$ & 0 & 0.32 \\
\hline Abdominal drain $+\mathrm{ABX}$ (No surgery) $-\mathrm{n}(\%)$ & 0 & $3(5 \%)$ & 0.08 \\
\hline $\mathrm{ABX}($ No surgery $)-\mathrm{n}(\%)$ & $3(5 \%)$ & $3(5 \%)$ & 0.95 \\
\hline Complicated Appendicitis—n (\%) & $13(21 \%)$ & $26(43 \%)$ & $<0.01$ \\
\hline \multicolumn{4}{|l|}{ Type of Complicated Appendicitis } \\
\hline Perforated Appendicitis-n (\%) & $7(11 \%)$ & $3(5 \%)$ & 0.22 \\
\hline Phlegmonous Appendicitis-n (\%) & $2(3 \%)$ & $8(13 \%)$ & 0.04 \\
\hline Localized pus-n (\%) & $1(2 \%)$ & $8(13 \%)$ & 0.02 \\
\hline Purulent peritonitis- $\mathrm{n}(\%)$ & $3(5 \%)$ & 0 & 0.08 \\
\hline Intra-operative Complications-n (\%) & $8(13 \%)$ & $14(24 \%)$ & 0.15 \\
\hline Bleeding-n $(\%)$ & $4(7 \%)$ & $4(7 \%)$ & 0.88 \\
\hline Spillage of bowel contents-n (\%) & $2(3 \%)$ & $2(4 \%)$ & 0.92 \\
\hline Other complications & $2(3 \%)$ & $7(11 \%)$ & 0.18 \\
\hline Abdominal Cultures taken-n (\%) & $6(10 \%)$ & $15(25 \%)$ & 0.03 \\
\hline Operation time-median(Range) & $45(15-147)$ & $46(20-173)$ & \\
\hline No. of Operations that took $\geq 90 \min -n(\%)$ & $4(7 \%)$ & $10(17 \%)$ & 0.1 \\
\hline
\end{tabular}

$A B X$ Antibiotics 
Table 3 PostOp complications and clinical outcome

\begin{tabular}{|c|c|c|c|}
\hline Characteristic & $\begin{array}{l}\text { March-April } 2019 \\
\text { group }(n=63)\end{array}$ & $\begin{array}{l}\text { March-April } 2020 \\
\text { group }(n=60)\end{array}$ & $P$ Value \\
\hline Length Of Stay (days) - mean \pm SD & $2.3 \pm 1.9$ & $2.6 \pm 2.1$ & 0.42 \\
\hline No. of Pts received PostOp ABX (IV/PO)—n (\%) & $20(32 \%)$ & $28(47 \%)$ & 0.09 \\
\hline PostOp Complications (SSI)—n (\%) & 0 & $2(4 \%)$ & 0.16 \\
\hline Readmission within 30 days $-\mathrm{n}(\%)$ & $3(5 \%)$ & $7(12 \%)$ & 0.17 \\
\hline \multicolumn{4}{|l|}{ Treatment on Readmission } \\
\hline $\mathrm{ABX}$ alone-n $(\%)$ & $3(5 \%)$ & $2(3 \%)$ & 0.69 \\
\hline Abdominal Drain $+\mathrm{ABX}-\mathrm{n}(\%)$ & 0 & $1(2 \%)$ & 0.32 \\
\hline Re-Laparoscopy-n (\%) & 0 & $3(5 \%)$ & 0.08 \\
\hline $\begin{array}{l}\text { No. of Pts } w / \text { Clavien-Dindo } \geq \text { grade } 2 \text { at } 2 \text { nd } \\
\text { Admission-n }(\%)\end{array}$ & 0 & $5(8 \%)$ & 0.02 \\
\hline Length Of Stay of 2nd Admission (days)—mean & 2.7 & 6 & \\
\hline Follow-up time (days) - mean \pm SD & $128 \pm 173$ & $31 \pm 51$ & $<0.01$ \\
\hline
\end{tabular}

Clavien-Dindo Classification for postoperative complications on the basis of the therapy used to correct a specific complication (I, II, IIIa, IIIb, IVa, IVb, and V)

IV/PO Intravenous/Per os, SSI Surgical Site Infection, Pts Patients, PostOp PostOperative, SSI Surgical Site Infection
Table 4 Logistic regression: risk factors for complicated appendicitis

\begin{tabular}{lllll}
\hline Factor & Sig. & OR & \multicolumn{2}{l}{$95 \%$ CI } \\
\cline { 3 - 5 } & & & Lower & Upper \\
\hline Age & 0.014 & 1.029 & 1.006 & 1.052 \\
BMI & 0.039 & 1.102 & 1.005 & 1.209 \\
$\begin{array}{l}\text { Pre-ED symptoms } \\
\text { duration (days) }\end{array}$ & 0.032 & 1.139 & 1.011 & 1.284 \\
\hline
\end{tabular}

$B M I$ Body mass index - weight in kilograms divided by the square of the height in meters, $E D$ Emergency Department

suggest a causative connection between the Lockdown and the severity of AA.

These findings are in accordance with a case series of seven children with a late diagnosis of complicated appendicitis resulting from a delay in seeking medical help due to fear of the global COVID-19 pandemic during the P20 [8]. Aviran et al. have evaluated the changes in patient's characteristics that came to the surgical ED during the Lockdown in general and have shown that patients were presented to the ED later in the course of their diseases and a worse clinical condition. Similar findings were reported worldwide regarding this period $[6,7,15,16]$.

Patient attendance at emergency departments around the world during the first wave of the COVID-19 pandemic declined dramatically [15-18]. This decline may be attributed to patient's trepidation of contracting the COVID-19 virus and disease at hospitals and trying to whether their illnesses by themselves at home.

Acute appendicitis is a prevalent surgical disease with a $7-8 \%$ lifetime risk of developing this condition $[19,20]$. The peak prevalence of the disease occurs at the ages of 10 to 25 and mainly during the spring and summer of the northern hemisphere [19, 21]. The definition of Complicated Appendicitis was described by Gomes et al. with a grading system of 0 to 5 based on laparoscopic findings [11]. Grades 3 and above are all considered complicated appendicitis. Grade 3 is necrotizing appendicitis, grade 4 is a peri-appendicular abscess, and grade 5 is diffuse peritonitis. Some of the known risk factors for complicated appendicitis are old age and the lengthy pre-treatment symptoms [22, 23]. As shown in the present study, in addition to the length of pre-ED symptoms duration, there was a direct correlation between age and BMI to the rate of complicated appendicitis, similar to prior reports.

The hypothesis that patients in P20 under the COVID-19 Lockdown had a higher prevalence of a more severe inflammatory condition compared to the correlating period of P19, beyond reports of a higher rate of complicated appendicitis findings in the operating room, is also strengthened by many other surrogate markers outlined in this study. For example, these markers were significantly elevated during P20 in periappendicular abscess and phlegmon findings on imaging, patients that were beyond surgery and received a percutaneous abdominal drain, the number of patients that surgical findings warranted an abdominal culture taken, the number of operations that took longer than $90 \mathrm{~min}$, and the number of patients that continued receiving postoperative antibiotics.

Although the incidence of complicated appendicitis and the average severity of inflammation were significantly higher in P20 than in P19, the patient's outcome was pretty similar with regard to the length of hospital stay. Although the rate of postoperative complications and 30 days readmission was higher in P20, the difference was not significant. In this entity, it is sound to believe that the reasons there 
were no short-term worse outcome are related to the fact that the patients were relatively young and healthy. Furthermore, acute appendicitis is mainly a localized inflammatory disease that, once removed, rarely has a lethal sequela. This might not be the case in elderly and morbidly obese patients as well as in patients with other emergent surgical conditions that may harbor worse long-term outcomes.

It is essential to state that none of the patients in this cohort suffered from the COVID-19 disease during their hospitalization. The limitations of this study lay in its retrospective nature and a single-center relatively modest size cohort, which may allow for a particular bias of the data. In addition, one should be careful to extrapolate conclusions to other regions in the world where the natural history of the pandemic might have evolved differently compared to Israel. Nonetheless, this study merits in highlighting the impact of treating the global COVID-19 pandemic with social restrictions on the non-COVID-19 ill patients and the possible added burden on patients and health care systems alike.

As more than a year of the COVID-19 pandemic has gone by and multiple outbreaks have been sighted in many countries worldwide, it is imperative to educate the public to seek medical help without delay as the price of delaying treatment might be grave.

\section{Conclusion}

Social restrictions such as lockdowns and "stay at home" orders, as used during the first outbreak of the COVID-19 pandemic, may have had an unfavorable impact on delaying patients from seeking timely medical help. This effect might lead patients to present to the ED in a more severe condition, as shown here for complicated appendicitis. The notion that widespread hard measures to treat one raging illness may result in harmful effects on patients suffering from other emergent diseases should be taken under consideration by health policymakers. Future outbreaks of COVID-19 or any other pandemics should warrant a push for educating the public to seek medical care when needed.

Funding None.

\section{Declarations}

Disclosures Dr. Yaron Rudnicki, Dr. Hagai Soback, Mr. Ori Mekiten, Dr. Guy Lifshiz, and Dr. Shmuel Avital declare no conflict of interest.

Informed consent For this type of study (retrospective in nature), formal consent is not required. An exemption from informed consent for this study was given by the institutional research committee as the data were retrospectively retrieved from an existing prospectively and routinely collected database.
Research involving human and animal rights This study has been approved by the appropriate institutional research committee (Approval No MMC-0223-20) and has been performed in accordance with the ethical standards as laid down in the 1964 Declaration of Helsinki and its later amendments or comparable ethical standards. ClinicalTrials. gov (ID - NCT04786041).

\section{References}

1. Wang C, Horby PW, Hayden FG, Gao GF (2020) A novel coronavirus outbreak of global health concern. Lancet 395(10223):470-473

2. Pullano G, Pinotti F, Valdano E et al (2020) Novel coronavirus (2019-nCoV) early-stage importation risk to Europe, January 2020. Eurosurveillance. https://doi.org/10.2807/1560-7917.ES. 2020.25.4.2000057

3. Kruchevsky D, Arraf M, Levanon S et al (2020) Trends in Burn Injuries in Northern Israel during the COVID-19 Lockdown. J Burn Care Res. https://doi.org/10.1093/jbcr/iraa154

4. Mast C, del Rio AM (2020) Delayed cancer screenings - a second look. Epic Health Research Network, Verona

5. Anteby R, Zager Y, Barash Y et al (2020) The impact of the coronavirus disease 2019 outbreak on the attendance of patients with surgical complaints at a tertiary hospital emergency department. J Laparoendosc Adv Surg Tech A 30(9):1001-1007

6. Aviran E, Laks S, Benvenisti $\mathrm{H}$ et al (2020) The impact of the COVID-19 pandemic on general surgery acute admissions and urgent operations: a comparative prospective study. Isr Med Assoc J 11(22):673-679

7. Justman N, Shahak G, Gutzeit O et al (2020) Lockdown with a price: the impact of the COVID-19 pandemic on prenatal care and perinatal outcomes in a tertiary care center. Isr Med Assoc J 22(9):533-537

8. Snapiri O, Rosenberg Danziger C, Krause I et al (2020) Delayed diagnosis of paediatric appendicitis during the COVID-19 pandemic. Acta Paediatr 109(8):1672-1676

9. Velayos M, Munoz-Serrano AJ, Estefania-Fernandez K et al (2020) Influence of the coronavirus 2 (SARS-Cov-2) pandemic on acute appendicitis. An Pediatr 93(2):118-122

10. Di Saverio S, Podda M, De Simone B et al (2020) Diagnosis and treatment of acute appendicitis: 2020 update of the WSES Jerusalem guidelines. World J Emerg Surg 15(1):27

11. Gomes CA, Nunes TA, Fonseca Chebli JM, Junior CS, Gomes CC (2012) Laparoscopy grading system of acute appendicitis: new insight for future trials. Surg Laparosc Endosc Percutan Tech 22(5):463-466

12. ICD-9-CM (1990) International classification of diseases, 9th revision, clinical modification. 3d edition, volumes 1,2 and 3 . Official authorized addendum effective October 1, 1990--HCFA. J Am Med Rec Assoc 61(8): suppl 1-35

13. Dindo D, Demartines N, Clavien PA (2004) Classification of surgical complications: a new proposal with evaluation in a cohort of 6336 patients and results of a survey. Ann Surg 240(2):205-213

14. Lee-Archer P, Blackall S, Campbell H et al (2020) Increased incidence of complicated appendicitis during the COVID-19 pandemic. J Paediatr Child Health 56(8):1313-1314

15. Jeffery MM, D'Onofrio G, Paek H et al (2020) Trends in emergency department visits and hospital admissions in health care systems in 5 states in the first months of the COVID-19 pandemic in the US. JAMA Intern Med 180(10):1328-1333

16. Tuominen J, Hallberg V, Oksala N et al (2020) NYU-EDA in modelling the effect of COVID-19 on patient volumes in a Finnish emergency department. BMC Emerg Med 20(1):97 
17. Cordoba M, Anteby R, Zager Y et al (2021) The effect of the COVID-19 outbreak on trauma-related visits to a tertiary hospital emergency department. Isr Med Assoc J 23(2):82-86

18. Willms AG, Oldhafer KJ, Conze S et al (2021) Appendicitis during the COVID-19 lockdown: results of a multicenter analysis in Germany. Langenbecks Arch Surg 406(2):367-375

19. Addiss DG, Shaffer N, Fowler BS, Tauxe RV (1990) The epidemiology of appendicitis and appendectomy in the United States. Am J Epidemiol 132(5):910-925

20. Mariage M, Sabbagh C, Grelpois G et al (2019) Surgeon's definition of complicated appendicitis: a prospective video survey study. Euroasian J Hepatogastroenterol 9(1):1-4

21. Fares A (2014) Summer appendicitis. Ann Med Health Sci Res $4(1): 18-21$
22. Naderan M, Babaki AE, Shoar S et al (2016) Risk factors for the development of complicated appendicitis in adults. Ulus Cerrahi Derg 32(1):37-42

23. Pham XD, Sullins VF, Kim DY et al (2016) Factors predictive of complicated appendicitis in children. J Surg Res 206(1):62-66

Publisher's Note Springer Nature remains neutral with regard to jurisdictional claims in published maps and institutional affiliations. 